\title{
SEMINAL, CLINICAL AND COLOR-DOPPLER ULTRASOUND CORRELATES OF PROSTATITIS-LIKE SYMPTOMS IN MALES OF INFERTILE COUPLES
}

Lotti F. ${ }^{1}$, Corona G. ${ }^{1,2}$, Mondaini N. ${ }^{3}$, Maseroli E. ${ }^{1}$, Rossi M. ${ }^{1}$ Filimberti E. ${ }^{1}$, Noci I. ${ }^{4}$, Forti G. ${ }^{1}$, , Maggi M. ${ }^{1}$

Sexual Medicine and Andrology Unit, Department, University of Florence, Florence, Italy'; Endocrinology Unit, Maggiore-Bellaria Hospital, Bologna, Italy²; Urology, Unit, Santa Maria Annunziata Hospital, Florence, Italy ${ }^{3}$ ${ }^{4}$ Department of Obstetrics and Gynecology, University of Florence, Florence, Italy

Introduction: Prostatitis-like symptoms (PLS) are common, often vague and not specific symptoms which may originate from the prostate or from other pelvic or scrotal organs.

-The National Institutes of Health-Chronic Prostatitis Symptom Index (NIH-CPSI) is nowadays considered the gold-standard instrument to assess PLS severity.

-So far, imaging of the prostate-vesicular region has been considered an "optional", poorly useful, tool in evaluating PLS.

-Although several studies previously investigated the impact of prostatitis, vesiculitis or epididymitis on semen parameters, the correlations between their related symptoms and scrotal/transrectal ultrasound characteristics have been poorly studied.

-No previous study systematically evaluated ultrasound correlates of PLS in men with couple infertility.

Aim: To investigate possible correlations between the NIH-CPSI total score and scrotal/transrectal colour-Doppler ultrasound (CDU) features and to evaluate the differences between subjects with and without PLS in a cohort of men with couple infertility.

Methods: PLS of 400 men (35.8 \pm 7.2 years) with couple infertility were assessed by the NIH-CPSI.

Prostatitis-like symptoms were defined by the presence of perineal and/or ejaculatory pain or discomfort and an NIH-CPSI pain score $\geq 4$, according to Nickel's criteria (Nickel et al., J Urol, 2001;165:842-5).

Symptoms were classified as "mild" or "moderate to severe" for an index pain score of 4 to 7 or $\geq 8$, respectively.

All patients underwent, during the same day, seminal analysis, including interleukin 8 (sIL-8), a reliable surrogate marker of prostatitis, and urine and seminal cultures, along with scrotal and transrectal CDU, before and after ejaculation.

Results PLS were detected in 39 (9.8\%) subjects, 17 (4.3\%) with "mild" and 22 (5.5\%) with "moderate to severe" symptoms.

$\square$ Prostatitis-like symptoms, $9.8 \%(\mathrm{n}=39)$
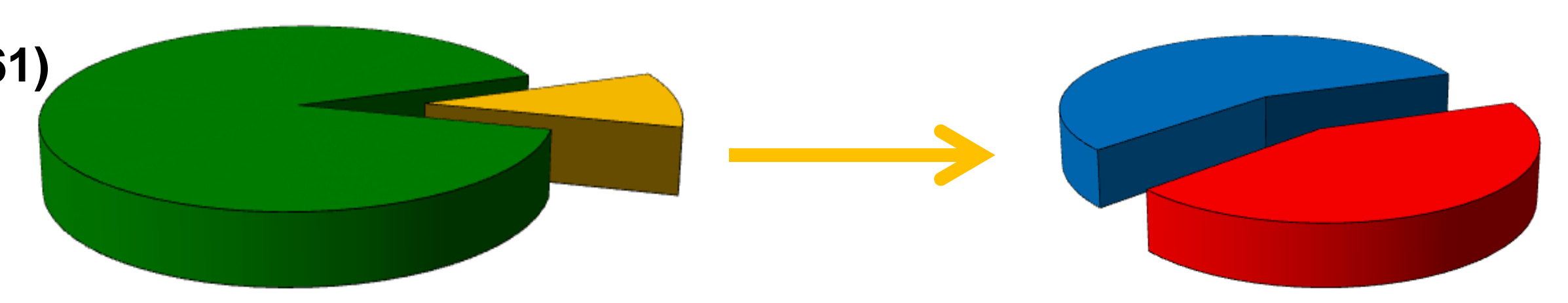

«mild» symptoms, $43.6 \%(n=17)$

$\square$ «moderate to severe» symptoms, $56.4 \%(n=22)$

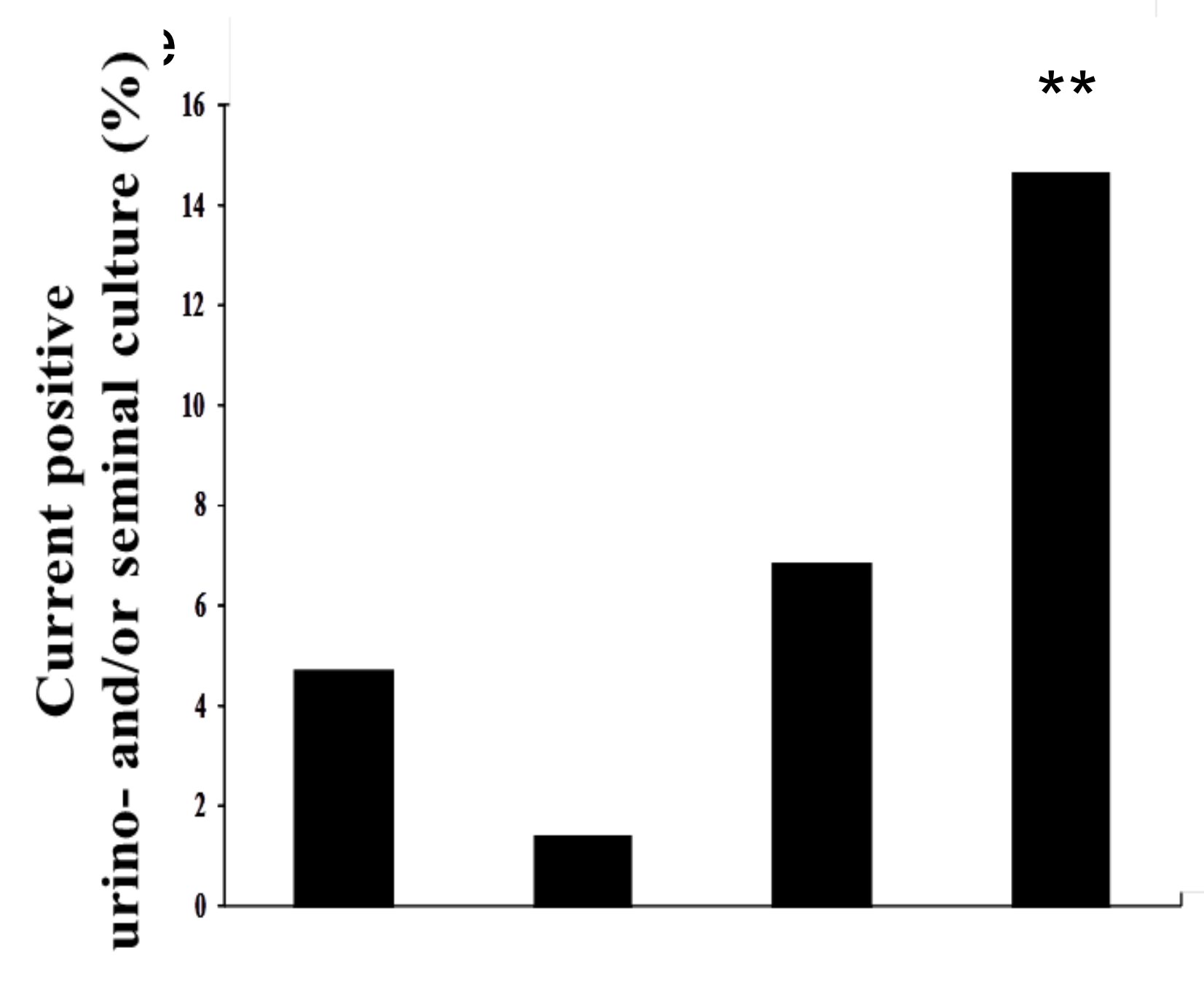

NIH-CPSI score (quartiles) Fig.1

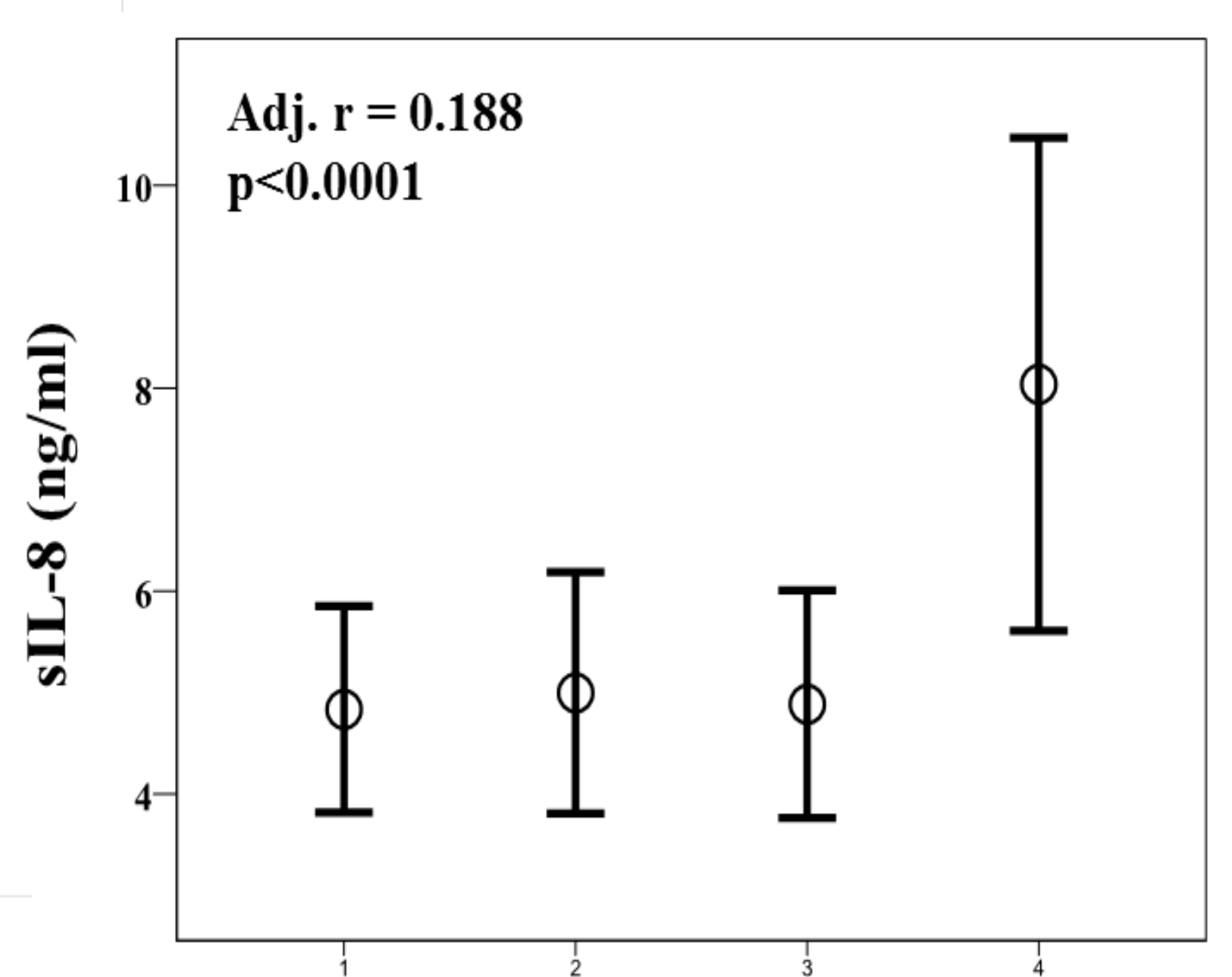

NIH-CPSI score (quartiles) Fig.2

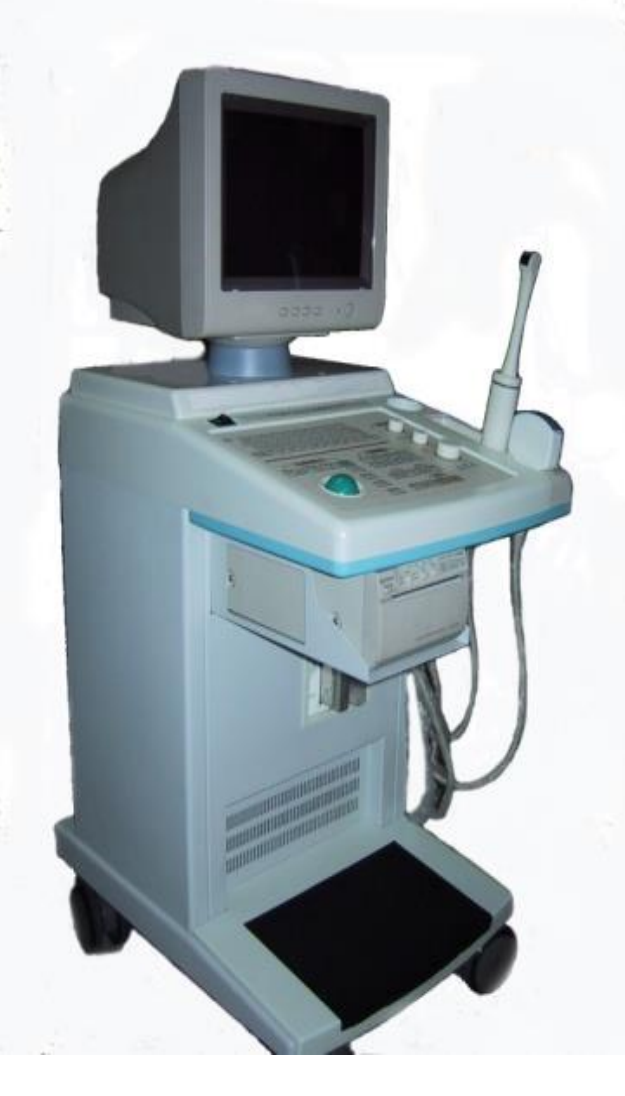

After adjusting for age, a positive association between $\mathrm{NIH}-\mathrm{CPSI}$ total and current positive urine and/or seminal cultures (Fig.1) and sIL-8 levels (Fig.2) was observed.

After adjusting for age, waist and total testosterone (TT), no association among NIH-CPSI (total or subdomain) scores or PLS and sperm parameters was observed.

${ }^{\star} p$ for trend 0.01
${ }^{\star \star} p$ for trend 0.001
${ }^{\star \star \star} p$ for trend 0.0001

In addition, subjects with a higher NIH-CPSI total score more often had CDU features suggestive of inflammation of the prostate (including inhomogeneity, Fig. 3; hypoechogenicity, Fig. 4; hyperaemia, Fig. 5 and a higher arterial prostatic peak systolic velocity, APPSV, Fig. 6), of the seminal vesicles and of the epididymis.

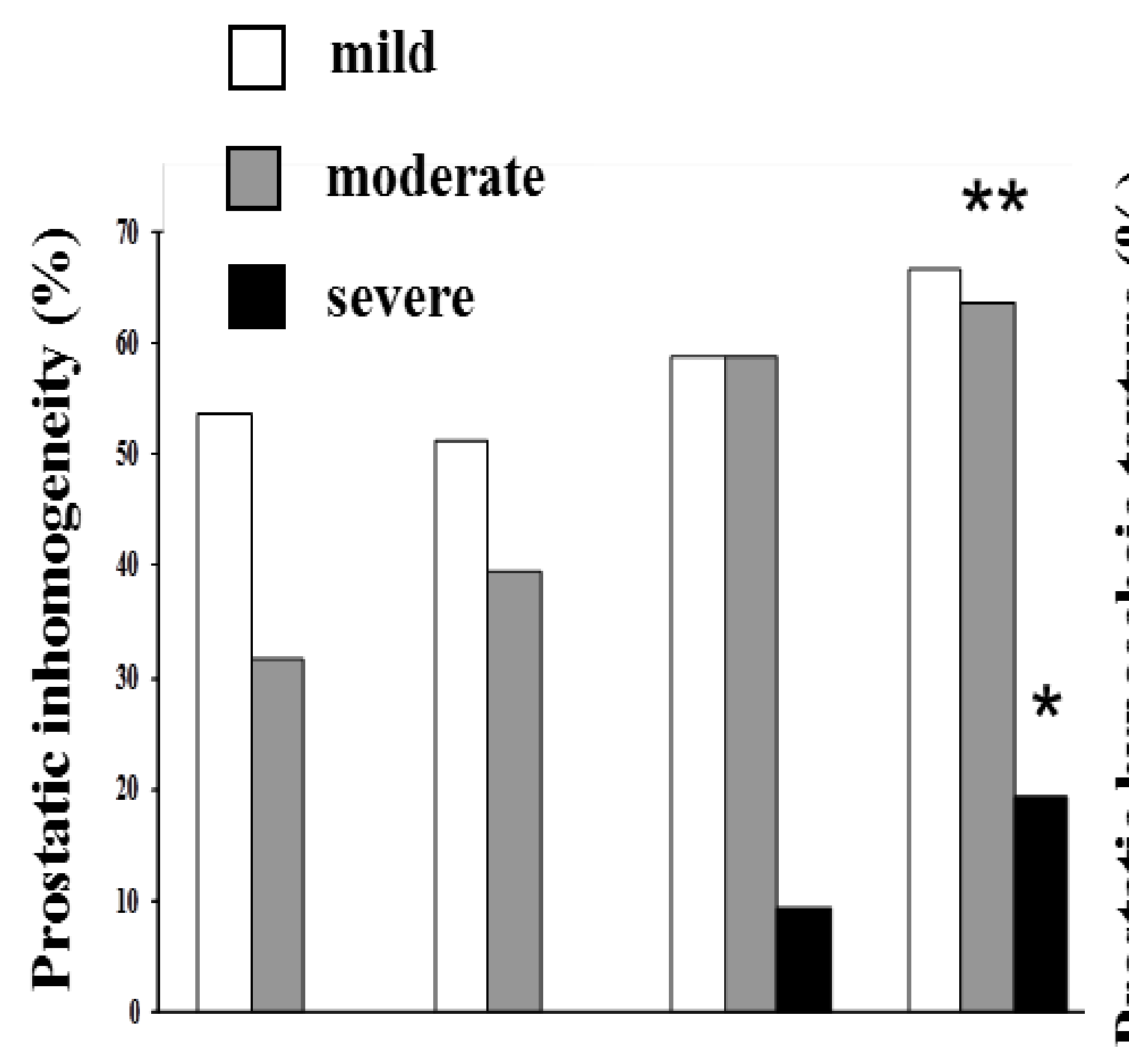

NIH-CPSI score (quartiles) Fig.3

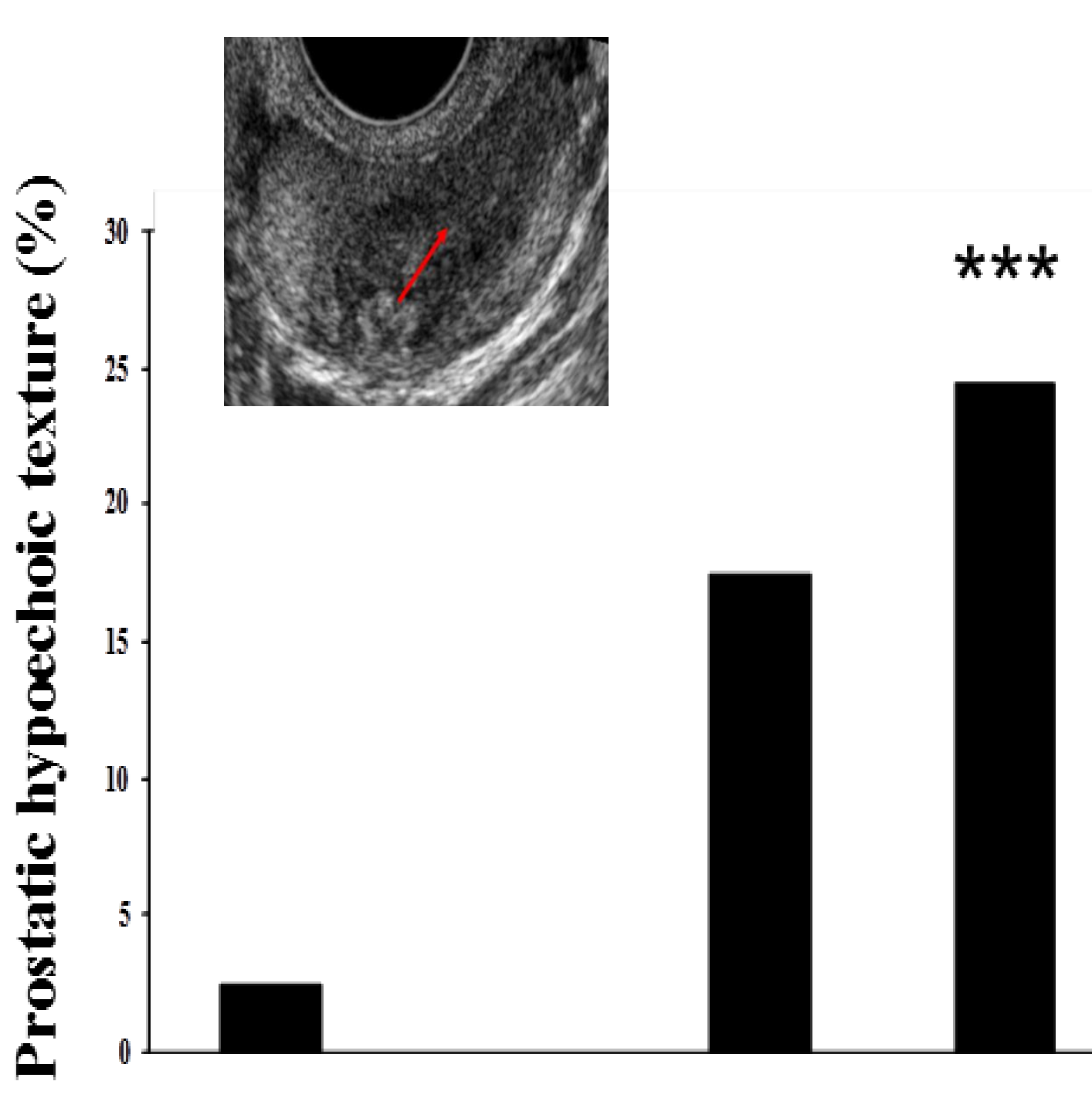

NIH-CPSI score (quartiles) Fig.4

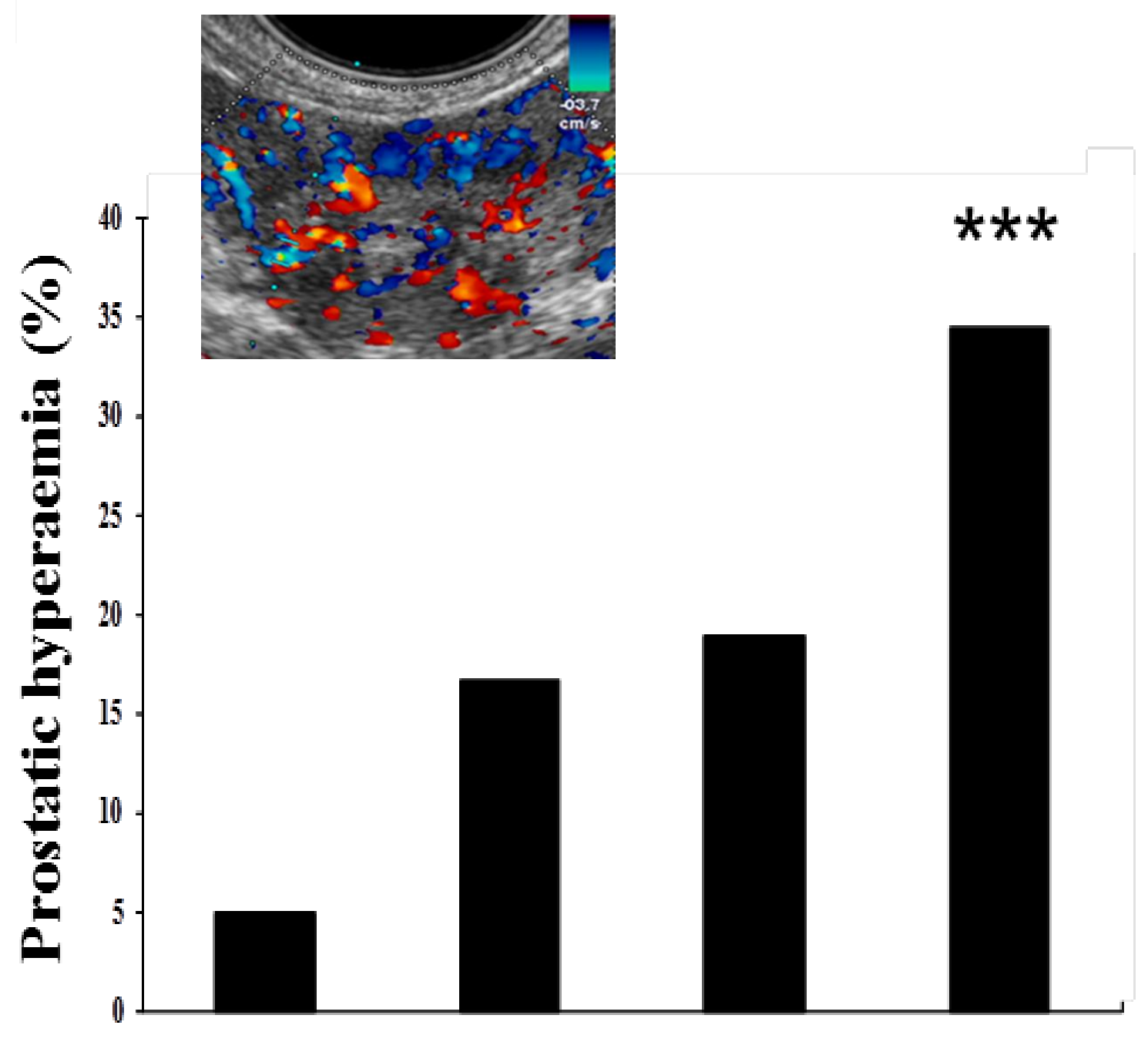

NIH-CPSI score (quartiles) Fig.5

When subjects with PLS were compared to the rest of the sample, they more often had a positive history of genito-urinary diseases, current positive urine and/or seminal cultures and higher sIL-8 levels, along with prostate-vesicular and epididymal abnormalities.

As assessed by receiver operating characteristic (ROC) curve, at $10.4 \mathrm{~cm} / \mathrm{sec}$ APPSV discriminates subjects with PLS with a specificity of $82 \%$ and sensitivity of $78 \%$, and an accuracy of $85.3 \pm 2.7 \%(p<0.0001)$ (Fig. 7).

When only subjects with PLS $(n=39)$ were investigated, those with moderate to severe symptoms had a higher prevalence of current positive urine and/or seminal cultures $(50 \% \mathrm{vs} 0 \%, \mathrm{p}<0.001)$.

The aforementioned significant associations of PLS were further confirmed by comparing PLS patients with age-, waist- and TT-matched PLS-free patients (1: 3 ratio).

\section{Conclusions:}

-NIH-CPSI total score is positively associated with positive urino and/or seminal cultures, higher sIL-8 levels, along with prostate-vesicular and epididymal abnormalities suggestive of inflammation.

-Patients with PLS have a higher prevalence of these abnormalities compared to the rest of the sample.

-We confirm APPSV as a parameter suggestive of prostate inflammation, and propose a $10.4 \mathrm{~cm} / \mathrm{sec}$ cut-off to predict PLS. Symptoms severity is mainly related to the presence of current positive cultures.

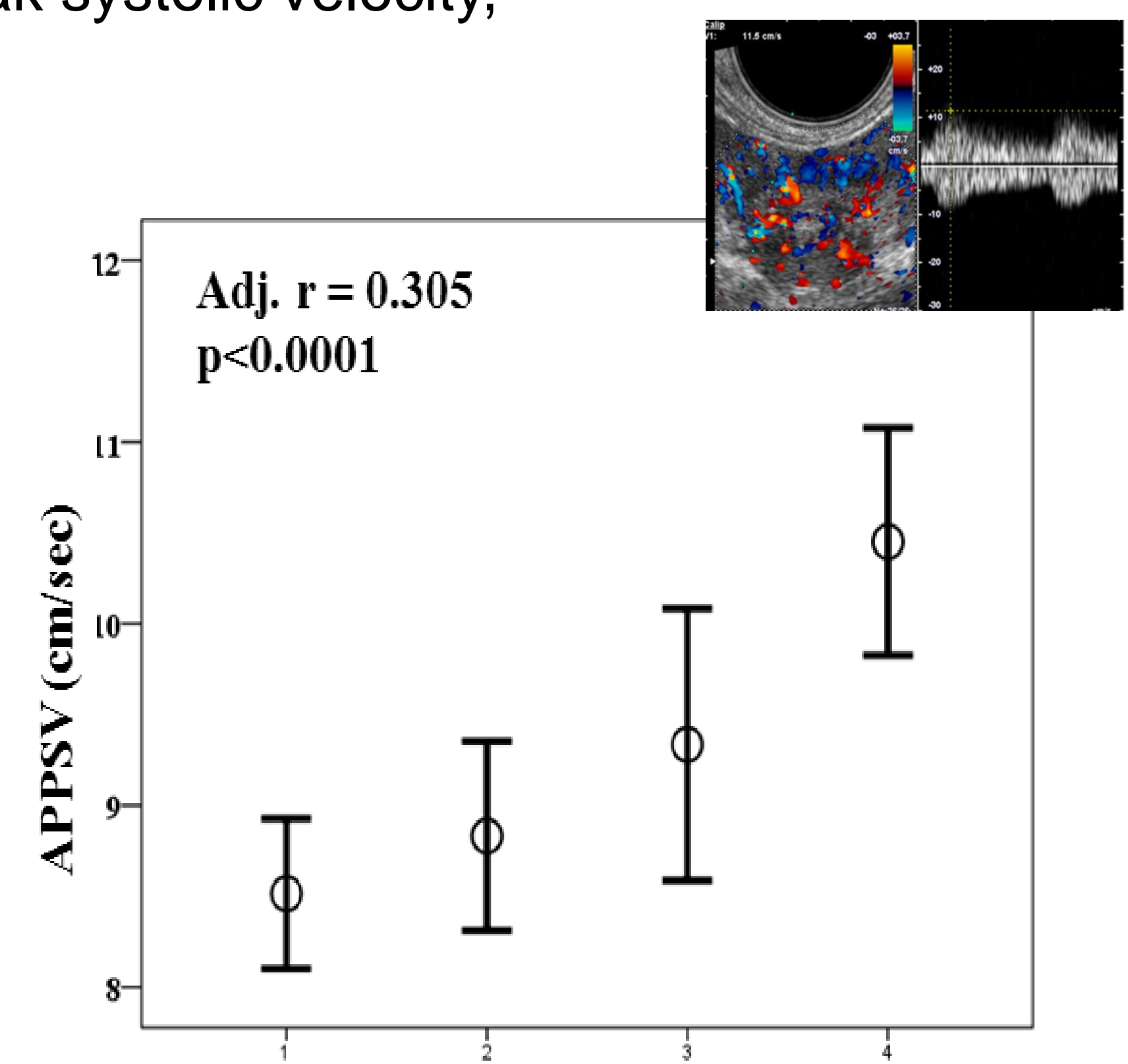

NIH-CPSI score Fig.6

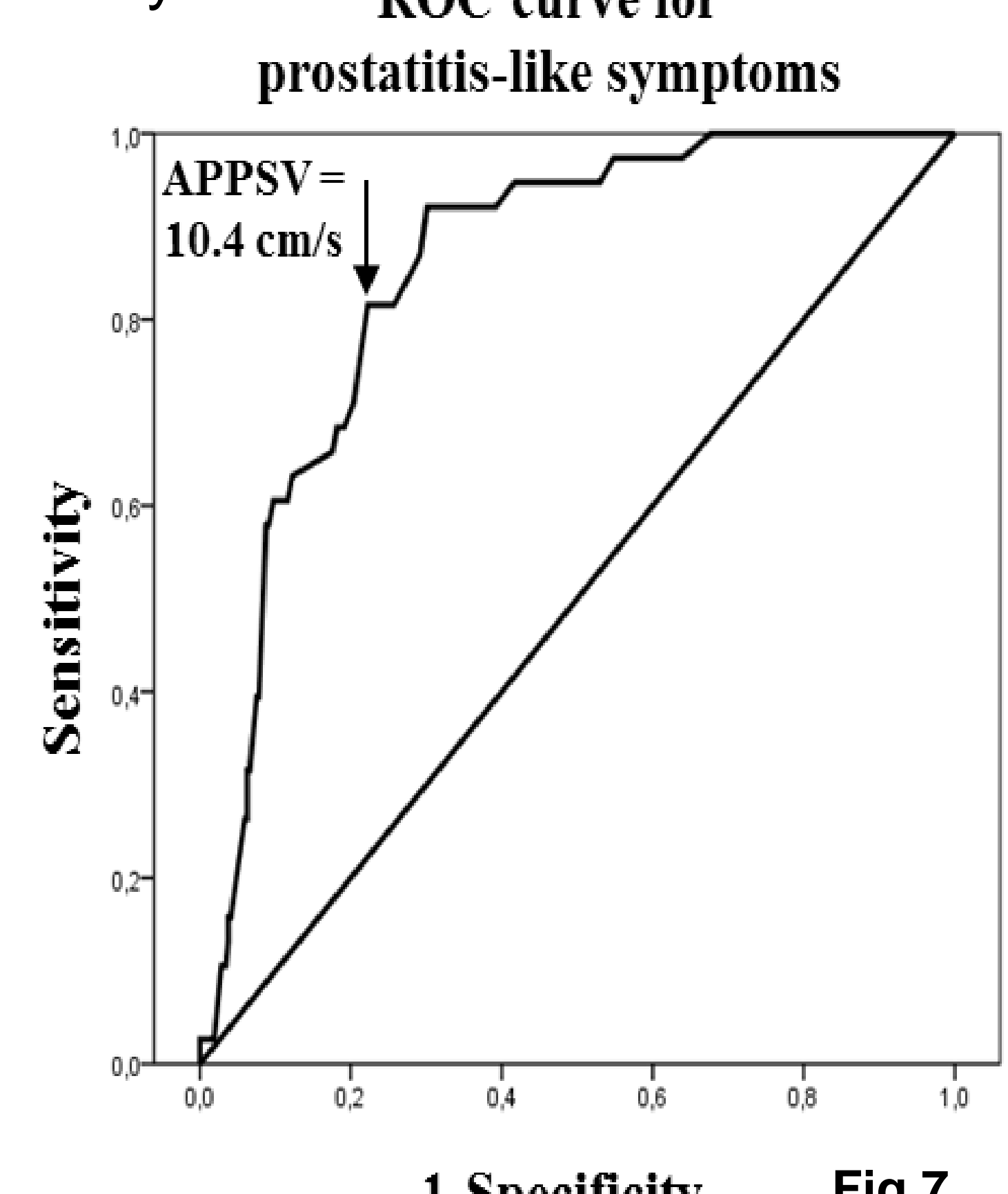

1-Specificity 